\title{
On the Determination of Rotational Line Half-Widths of Diatomic Molecules*
}

\author{
S. S. Penner and H. S. Tsien \\ Guggenheim Jet Propulsion Center, California Institute of Technology, Pasadena, California
}

(Received January 21, 1952)

\begin{abstract}
A simple closed-form expression is obtained for the fractional intensity of radiation absorbed by vibrationrotation bands with collision-broadened spectral lines. The resulting expressions greatly reduced the labor involved in obtaining apparent rotational half-widths from experimental measurements.
\end{abstract}

\section{INTRODUCTION}

A $\mathrm{T}$ room temperature the integrated absorption of rotational lines is given, in adequate approximation, by the relations ${ }^{1,2}$

$$
\begin{aligned}
& S_{j \rightarrow j-1}{ }^{0 \rightarrow 1}=\alpha_{F}\left(\omega_{j \rightarrow j-1}{ }^{0 \rightarrow 1} / \omega^{*}\right)[(j-\lambda)(j+\lambda) / j] \\
& \times[\exp -E(0, j) / k T] Q^{-1}, \\
& S_{j-1 \rightarrow j^{0 \rightarrow 1}}=\alpha_{F}\left(\omega_{j-1 \rightarrow j^{0 \rightarrow 1}} / \omega^{*}\right)[(j-\lambda)(j+\lambda) / j] \\
& \times[\exp -E(0, j-1) / k T] Q^{-1}, \\
& S_{j \rightarrow j}^{0 \rightarrow 1}=\alpha_{F}\left(\omega_{j \rightarrow j}^{0 \rightarrow 1} / \omega^{*}\right)\left[\lambda^{2}(2 j+1) / j(j+1)\right] \\
& \times[\exp -E(0, j) / k T] Q^{-1}, \\
& S_{j \rightarrow j^{\prime}}, 0 \rightarrow 2 / S_{j \rightarrow j^{\prime}}, 0 \rightarrow 1=\alpha_{0} / \alpha_{F} .
\end{aligned}
$$

Here $\alpha_{F}$ and $\alpha_{0}$ represent, respectively, the integrated absorption of the fundamental and first overtone; $\omega_{j \rightarrow j^{\prime}} \rightarrow 1$ is the wave number corresponding to the rotational transition $j \rightarrow j^{\prime}$ and the vibrational transition $0 \rightarrow 1 ; \omega^{*}=$ wave number for the transition $j=0 \rightarrow j=0$, $n=0 \rightarrow n=1 ; \lambda=$ quantum number measuring the component of electronic angular momentum about the internuclear axis; $E(0, j)=$ energy of the zeroth vibrational and $j$ 'th rotational level; $k=$ Boltzmann constant; $T=$ absolute temperature; $Q=$ complete partition function.

In an absorption experiment with a source of spectral radiant intensity $R_{s \omega}$, the fractional absorbed intensity $R_{a \omega} / R_{s \omega}$, integrated over an entire vibration-rotation band of width $\Delta \omega_{B}$, is

$$
\int_{\Delta \omega B}\left[\left(R_{\alpha \omega} / R_{s \omega}\right)\right] d \omega=\int_{\Delta \omega_{B}}\left[1-\exp \left(-P_{\omega} X\right)\right] d \omega,
$$

where $P_{\omega}$ represents the spectral absorption coefficient and $X$ is the optical density. For collision-broadened spectral lines which do not overlap ${ }^{3,4,5}$

$$
\int_{\Delta \omega_{B}}\left(R_{a \omega} / R_{s \omega}\right) d \omega=\sum_{j} 2 \pi b_{j \rightarrow j^{\prime}}, \rightarrow \rightarrow 1\left[f\left(x_{j \rightarrow j^{\prime}}{ }^{0 \rightarrow 1}\right)\right],
$$

* Supported, in part, by the ONR under Contract Nonr-220(03), NR 015210.

${ }^{1}$ S. S. Penner, J. Chem. Phys. 19, 272, 1434 (1951).

2 B. L. Crawford, Jr., and H. L. Dinsmore, J. Chem. Phys. 18 983, $1682(1950)$

${ }^{3}$ S. S. Penner and D. Weber, J. Chem. Phys. 19, 1351, 1361 (1951).

${ }^{4}$ R. Ladenburg and F. Reiche, Ann. Physik 42, 181 (1913).

${ }^{5}$ W. M. Elsasser, Harvard Meteorological Studies No. 6 (Blue Hill Observatory, Milton, Massachusetts, 1942). where

$$
2 \pi b_{j \rightarrow j^{\prime}}, 0 \rightarrow 1\left[f\left(x_{j \rightarrow j^{\prime}}, \rightarrow 1\right)\right]
$$$$
\simeq 2\left(S_{j \rightarrow j^{\prime}}{ }^{0 \rightarrow 1} b_{j \rightarrow j^{\prime}}{ }^{0 \rightarrow 1} X\right)^{\frac{1}{3}} \text { for large values of } x_{j \rightarrow j^{\prime}}{ }^{0 \rightarrow 1}(5 \mathrm{a})
$$

and

$$
\begin{aligned}
2 \pi b_{j \rightarrow j^{0}},{ }^{0 \rightarrow 1} & {\left[f\left(x_{j \rightarrow j^{\prime}}, 0 \rightarrow 1\right)\right] } \\
& \simeq S_{j \rightarrow j^{\prime}}{ }^{0 \rightarrow 1} X \text { for small values of } x_{j \rightarrow j^{\prime}}{ }^{0 \rightarrow 1} .
\end{aligned}
$$

The symbol $b_{j \rightarrow j^{\prime}, 0 \rightarrow 1}$ denotes one-half of the spectral half-width for the indicated transition. Equations (5a) and (5b) represent asymptotic limiting forms in which the right-hand sides exceed the value of $2 \pi b_{j \rightarrow j^{0}}{ }^{0 \rightarrow 1}$ $\times\left[f\left(x_{j \rightarrow j^{\prime}} \rightarrow 1\right)\right]$ for every value of $x_{j \rightarrow j^{\prime}} \rightarrow 1$. Equation (5a) constitutes a better approximation for $x_{j \rightarrow j^{\prime}}{ }^{0 \rightarrow 1}>(2 / \pi)$, whereas Eq. (5b) is preferable for $x_{j \rightarrow j^{\prime}} \rightarrow \rightarrow 1<(2 / \pi)$. Since, at room temperature, $\alpha_{F}=\sum_{j} S_{j \rightarrow j^{\prime}}{ }^{0 \rightarrow 1}$, it is evident from Eqs. (4) and (5b) that

$$
\int_{\Delta \omega B}\left(R_{a \omega} / R_{s \omega}\right) d \omega=\alpha_{F} X, \text { for small values of } x_{j \rightarrow j^{\prime}}, \rightarrow \rightarrow 1 .(6)
$$

Equation (6) is an illustration of the well-known result that accurate measurements of $\alpha_{F}$ can be obtained for very small values of the optical density. It is evident that under these conditions the experimental results are independent of the line width.

\section{CALCULATION OF ROTATIONAL HALF-WIDTHS FROM EXPERIMENTAL DATA}

In order to obtain a useful procedure for the calculation of rotational half-widths, it is necessary to assume that $b_{j \rightarrow j^{\prime}}{ }^{0 \rightarrow 1}=b_{F}, b_{j \rightarrow j^{\prime}}{ }^{0 \rightarrow 2}=b_{0}$ and to evaluate the righthand side of Eq. (4) by using Eqs. (5a) and (1a) to (2). We shall outline the procedure for the fundamental vibration-rotation band.

Equations (1a) to (1c), (4), and (5a) lead to the relation

$$
\begin{aligned}
\int_{\Delta \omega_{F}} & \left(R_{a \omega} / R_{s \omega}\right) d \omega \\
= & 2\left\{\alpha_{F} b_{F} X / Q \exp [E(0,0) / k T]\right\}^{\frac{1}{3}} \\
& \times \sum_{j}[(j-\lambda)(j+\lambda) / j]^{\frac{3}{2}}\left\{\left(\omega_{j \rightarrow j-1}^{0 \rightarrow 1} / \omega^{*}\right)^{1}\right. \\
& \times\{\exp -[E(0, j)-E(0,0)] / 2 k T\}+\left(\omega_{j-1 \rightarrow j^{0 \rightarrow 1}} / \omega^{*}\right)^{\frac{1}{2}} \\
& \times\{\exp -[E(0, j-1)-E(0,0)] / 2 k T\}\} \\
& +\lambda[(2 j+1) / j(j+1)]^{\frac{1}{2}}\left(\omega_{j \rightarrow j^{0 \rightarrow 1}} / \omega^{*}\right)^{\frac{1}{2}} \\
& \quad \times\{\exp -[E(0, j)-E(0,0)] / 2 k T\} . \quad(7)
\end{aligned}
$$


Equation (7) is the basic equation from which useful results can be obtained for two special cases.

\section{A. Diatomic Molecules Without $Q$-Branch $(\lambda=0)$}

For diatomic molecules without $Q$-branch $(\lambda=0)$ Eq. (7) becomes

$$
\begin{aligned}
\int_{\Delta \omega_{F}}\left(R_{a \omega} / R_{s \omega}\right) d \omega \\
=2\left\{\alpha_{F} b_{F} X / Q \exp [E(0,0) / k T]\right\}^{\frac{1}{3}} \\
\quad \times \sum_{j} j^{\frac{1}{2}}\left\{\left(\omega_{j \rightarrow j-1} 0 \rightarrow 1 / \omega^{*}\right)^{\frac{3}{3}}\right. \\
\quad \times \exp -[E(0, j)-E(0,0)] / 2 k T+\left(\omega_{\left.j-1 \rightarrow j^{0 \rightarrow 1} / \omega^{*}\right)^{\frac{1}{2}}}\right. \\
\quad \times \exp -[E(0, j-1)-E(0,0)] / 2 k T\} . \quad(7 \mathrm{a}
\end{aligned}
$$

Utilizing customary spectroscopic notation ${ }^{6}$ and expanding the various terms in Eq. (7a) in powers of $\gamma j$, it is found that, correct to powers of $\gamma^{2} j^{2},{ }^{7}$

$$
\begin{gathered}
\int_{\Delta \omega_{F}}\left(R_{a \omega} / R_{s \omega}\right) d \omega=2\left\{\alpha_{F} b_{F} X / Q \exp [E(0,0) / k T]\right\}^{\frac{1}{2}} \\
\text { where } \quad \times \sum_{j^{2}} 2 j^{2}\left[\exp -\left(\gamma u j^{2} / 2\right)\right]\left[1+\gamma^{2} j^{2} h(\gamma, \delta, u)\right], \\
h(\gamma, \delta, u)=\left(u^{2} / 8\right)+(u / 2)-[(\delta / \gamma)+1] / 2 .
\end{gathered}
$$

The sum over $j$ can be evaluated readily by use of the Euler-Maclaurin summation formula. ${ }^{7}$ In this manner it is found that, in good approximation,

$$
\int_{\Delta \omega_{F}}\left(R_{a \omega} / R_{s \omega}\right) d \omega=4.1(2)\left(\alpha_{F} b_{F} X\right)^{\frac{1}{2}}(\gamma u)^{-\frac{1}{2}}
$$

where a suitable expansion has been introduced ${ }^{6}$ for $\{Q \exp [E(0,0) / k T]\}^{\frac{1}{2}}$. The error in Eq. (8) is estimated to be of the order of $(\gamma u)^{3}$. It is evident that Eq. (8) holds for the first overtone with $\alpha_{F}$ and $b_{F}$ replaced by $\alpha_{0}$ and $b_{0}$, respectively.

\section{B. Diatomic Molecules With Q-Branch $(\lambda \neq 0)$}

In the general case $\lambda \neq 0$ we must proceed from Eq. (7) directly. After suitable expansion of various quantities appearing in Eq. (7) it is found that

$$
\begin{aligned}
\int_{\Delta \omega F}( & \left.R_{a \omega} / R_{s \omega}\right) d \omega=2\left(\alpha_{F} b_{F} X\right)^{\frac{1}{2}}(\gamma u)^{+\frac{1}{2}} \\
& \times \sum_{j}\left\{2[(j-\lambda)(j+\lambda) / j]^{\frac{1}{2}}\right. \\
& \left.\times\left[\exp \left(-\gamma u j^{2} / 2\right)\right]\left[1+\gamma^{2} j^{2} h(\gamma, \delta, u)\right]\right\} \\
+ & \left\{\lambda[(2 j+1) / j(j+1)]^{\frac{1}{2}}\left[\exp \left(-\gamma u j^{2} / 2\right)\right]\right. \\
& \left.\times\left[1-(u \gamma j / 2)+\left(u^{2} \gamma^{2} j^{2} / 8\right)\right]\right\}
\end{aligned}
$$

${ }^{6}$ J. E. Mayer and M. G. Mayer, Statistical Mechanics (John Wiley and Sons, Inc. New York, 1940).

${ }^{7}$ For details, describing a similar evaluation, see Penner, Ostrander, and Tsien, J. Appl. Phys. 23, 256 (1952).
Since $\lambda$ is generally a small integer and since the contributions made to the value of the sum by rotational lines with small values of $j$ are relatively small, we may approximate $[(j-\lambda)(j+\lambda) / j]^{\frac{1}{2}}$ by $j^{\frac{1}{2}}$, whence it follows that the first brace of the sum in Eq. (7b) again leads to the result given in Eq. (8). Thus it is only necessary to estimate the total contributions made by the $Q$-branch. An adequate approximation is obtained by utilizing again the Euler-Maclaurin summation formula. The result is

$$
\begin{aligned}
\int_{\Delta \omega F}\left(R_{a \omega} / R_{s \omega}\right) d \omega \\
\quad=4.1(2)\left(\alpha_{F} b_{F} X\right)^{\frac{1}{2}}(\gamma u)^{-\frac{3}{2}}\left[1+1.4(8) \lambda(\gamma u)^{\frac{1}{2}}\right] .
\end{aligned}
$$

The error in Eq. (9) is also of the order of $(\gamma u)^{\frac{3}{2}}$. Equation (9) applies to the first overtone if $\alpha_{F}$ and $b_{F}$ are replaced by $\alpha_{0}$ and $b_{0}$, respectively.

Because of very close spacing of the rotational lines in the $Q$-branch, the right-hand side of Eq. (9) will generally be too large. For this reason it may be indicated to use Eq. (9) in the form

$$
\begin{aligned}
& \int_{\Delta \omega F}\left(R_{a \omega} / R_{8 \omega}\right) d \omega \\
& \quad=4.1(2)\left(\alpha_{F} b_{F} X\right)^{\frac{1}{2}}(\gamma u)^{-\frac{1}{2}}\left[1+1.4(8) \lambda \beta(\gamma u)^{\frac{1}{2}}\right]
\end{aligned}
$$

where $\beta$ is an empirically determined correction factor which is less than or equal to unity. The contribution of the $Q$-branch is usually so small that it may be neglected in the treatment of experimental data.

In conclusion, we wish to emphasize again that the results which we have obtained are useful only if the Lorentz dispersion formula is adequate to describe the experimental measurements. This condition seems to apply to simple diatomic molecules such as $\mathrm{CO}$ and $\mathrm{NO}$ but not to $\mathrm{HCl}$ and $\mathrm{HBr}^{3,8}$ If the empirical data obtained cannot be correlated quantitatively by expressions of the form given in Eqs. (8) and (9), then it is generally safe to assume that an oversimplified description of spectral line-shape has been used. ${ }^{9}$

${ }^{8}$ S. S. Penner and D. Weber, paper presented before the Symposium on Molecular Structure and Spectroscopy (Ohio State University, Columbus, Ohio, 1951).

${ }_{9}^{9}$ In this connection reference should be made to the extensive literature on pressure broadening of spectral lines, for example, H. A. Lorentz, Proc. Amst. Akad. Sci. 8, 59 (1906); W. Lenz, Z. Physik 80, 423 (1933); V. F. Weisskopf, Physik Z. 34, 1 (1933); E. Lindholm, Arkiv. Mat. Astron. Fysik. 32, 17 (1945); J. H. Van Vleck and V. F. Weisskopf, Revs. Modern Phys. 17, 227 (1945); J. H. Van Vleck and H. Margenau, Phys. Rev. 76, 1211 (1949) ; P. W. Anderson, Phys. Rev. 76, 647 (1949); H. Margenau, Phys. Rev. 82, 156 (1951). 